\title{
UNDERGROUND TUNNELS DETECTION AND LOCATION BY HIGH RESOLUTION SEISMIC REFLECTION
}

\author{
B.PIWAKOWSKI, J.C.TRICOT, C.LEONARD, N.OUARRADI,B.DELANNOY \\ Groupe Electronique-Acoustique EC-Lille (IEMN DOAE UMR 9929CNRS), Ecole Centrale de \\ Lille, 59651 Villeneuve d'Ascq, BP48, France.
}

\section{INTRODUCTION}

In spite of the widespread development of high-resolution seismic reflection techniques during recent years, the detection of underground cavities remains fundamentally unsolved. Except for some general principles, each case requires a particular study. Until now, the successful location of small (in regard to wavelength) cavities has been performed in an indirect way, i.e. by means of the detection of the cavity-induced perturbations of the seismic image of the regular geological structure (which should be known in advance) [1],[2],[3],[4] The direct detection of cavities by means of the reflection originating from the top of a void, though theoretically possible, gives no results in practice.[5]. This paper presents the successful application of the high resolution seismic reflection for the indirect detection and location of abandoned old chalk-pits, appearing in the north of France and being now unexploited. Some positions of these voids which form a network of tunnels are mapped, whereas others are unknown and represent a real danger for both population and building activities. The the tunnels occur typically in chalk at a depth of 8-15 m and are followed by a water table and a marl layer (Fig.1).

\section{PROCEDURE}

Localisation is performed indirectly by detection of the gallery-induced perturbations in the regular seismic pattern. The detection procedure includes two stages:

(1).The high resolution CDP prospection of the profile respecting the basic principles of this technique This stage reveals two principal markers: the water table (B) and the interface chalk/marl (C). Acquisition is carried out using the 24-channel EGG2401 seismograph and 100 $\mathrm{Hz}$ geophones. which provide the low-cut filtering of the seismic data. Particular care is taken to make the correct choice of the recording geometry in regard to the markers depths [6]. A silenced "betsy gun" [7] (designed in the laboratory) firing 12-gauge hunting cartridges, is used as a source. It performs well for depths of our interest and enables operation directly at the surface, thus avoiding the drilling of shot-holes. The obtained exploitable wavelet varies from $200 \mathrm{Ho} 250 \mathrm{~Hz}$ assuring sufficient depth resolution and the cavity dimension/wavelength ratio no poorer then 0.5 .

(2). Interpretation and classification of the direct and indirect signs of the cavity occurrence. In fact, the reflectors B and $C$ which represent quasi-stationary well correlated events can be used as the reference signals for the observation of the abnormal indirect signs. The numerous experiments conducted at the areas with known cavity positions allowed us to construct the "cavity symptoms list" including all the signs correlated with the cavity occurrence. These symptoms are respectively (see example of Fig.2): amplitude perturbation of $B$, amplitude drop of the high frequency version of $B$, spatial correlation drop of $B$, spectral modifications of $B$, masking of reflector $C$, spatial correlation drop of $C$. In most of situations the masking 
of reflector C (see Fig.2.c) is so clearly seen that cavity can be interpreted directly from the seismic section. In other situations, these signs are poorer, thus to improve the reliability of detection, they should be considered together. In order to help the interpretation all symptoms are expressed in binary scale scale $(0-1)$ and their weighted sum .is assumed to be equal to the risk of the cavity presence (CR). This signal may be plotted directly as a function of CDP, enabling thus an easy interpretation and presentation of the results. The example of six seismic sections processed in order to obtain the evaluation of the cavity induced symptoms, is shown in Fig.2). The successful aplication of the procedure is presented in Fig.3

\section{CONCLUSIONS}

For the given geological conditions, the high resolution seismic may be useful for the location of chalk mines. The proposed "indirect" approach gives very good results in practical applications. The borehole tests confirm $80 \%$ of detections (since the cavities never occupy more than about $75 \%$ of the surface, these results can be considered as very favourable). As well, the precision of detection of the depth of void is better than $15 \%$. We point out that the presented approach may not be interpreted as the "artificial inteligence" applied to detection of the cavities. The human interpretation is here the substantial factor.

\section{REFERENCES}

[1] Alleman, G., 1996. High resolution seismic profiling applied to a heterogeneous area imaging and to cavity detection in Switzerland. Ph.D. dissertation, Université de Lausanne, [2] Branham, K.L. and Steeples, D.W., 1988. Cavity detection using high-resolution seismic reflection methods. Mining Engineering, February: 115-119.

[3] Kourkafas, P., Goulty, N.R., 1996. Seismic reflection imaging of gypsum mine working at Sherburn-in-Elmet, Yorkshire, England..European Journal of Environmental and Engineering Geophysics, 1: 53-63.

[4] Piwakowski, B., Tricot, J. C. and Delannoy, B., 1994. Underground tunnels detection and location by high resolution seismic reflection. Proceedings of 56 th International Symposium of European Association of Exploration Geophysicists, Vienna, June 6-10.

[5] Piwakowski B, Watelet J.C, Moreaux D, Sbai K. " High resolution seismic prospection of old gypsum mines-evaluation of detection possibilities" Proceedings 2nd Congress of Environmental \& Engineering Geophysical Society, Nantes, France.

[6] Knapp, R.W., Steeples, D.W., 1986. High resolution common-depth-point seismic reflection profilling: field acquisition parameter design. Geophysics, 51: 283-294.

[7] Miller, R.D., Pullan,S.E., Waldner,J.S. and Haeni, F.P., 1986. Field comparison of seismic sources. Geophysics, 51: 2067-2092.

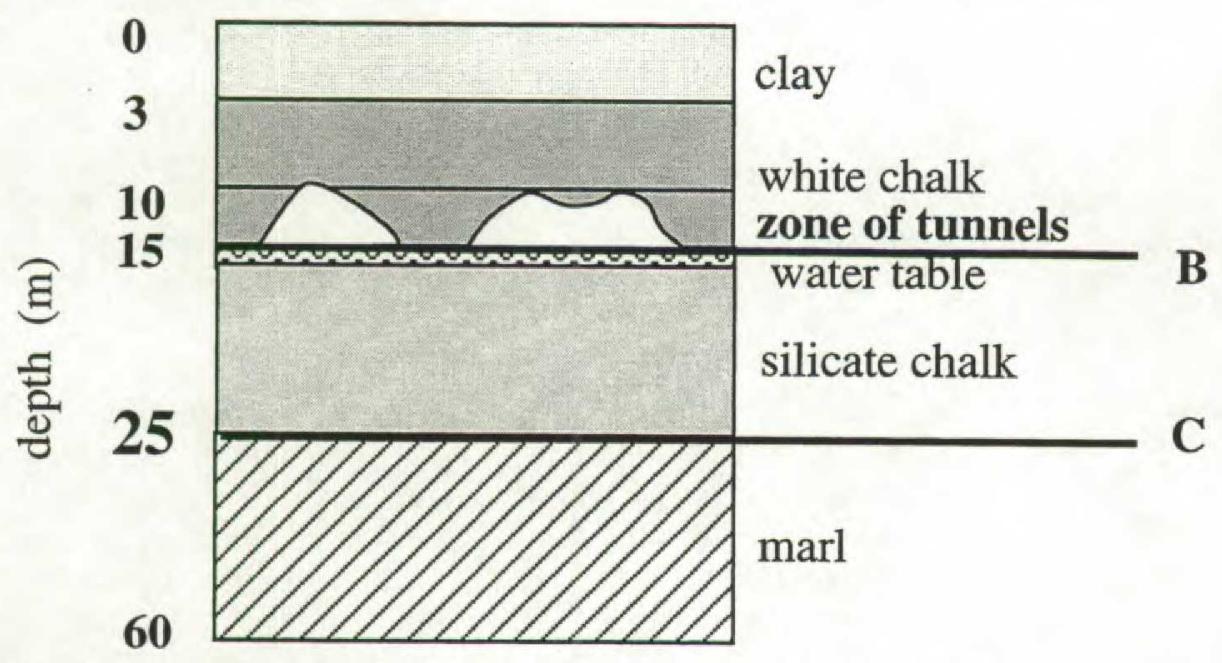

Fig. 1 General geological data in the region of experiments. 


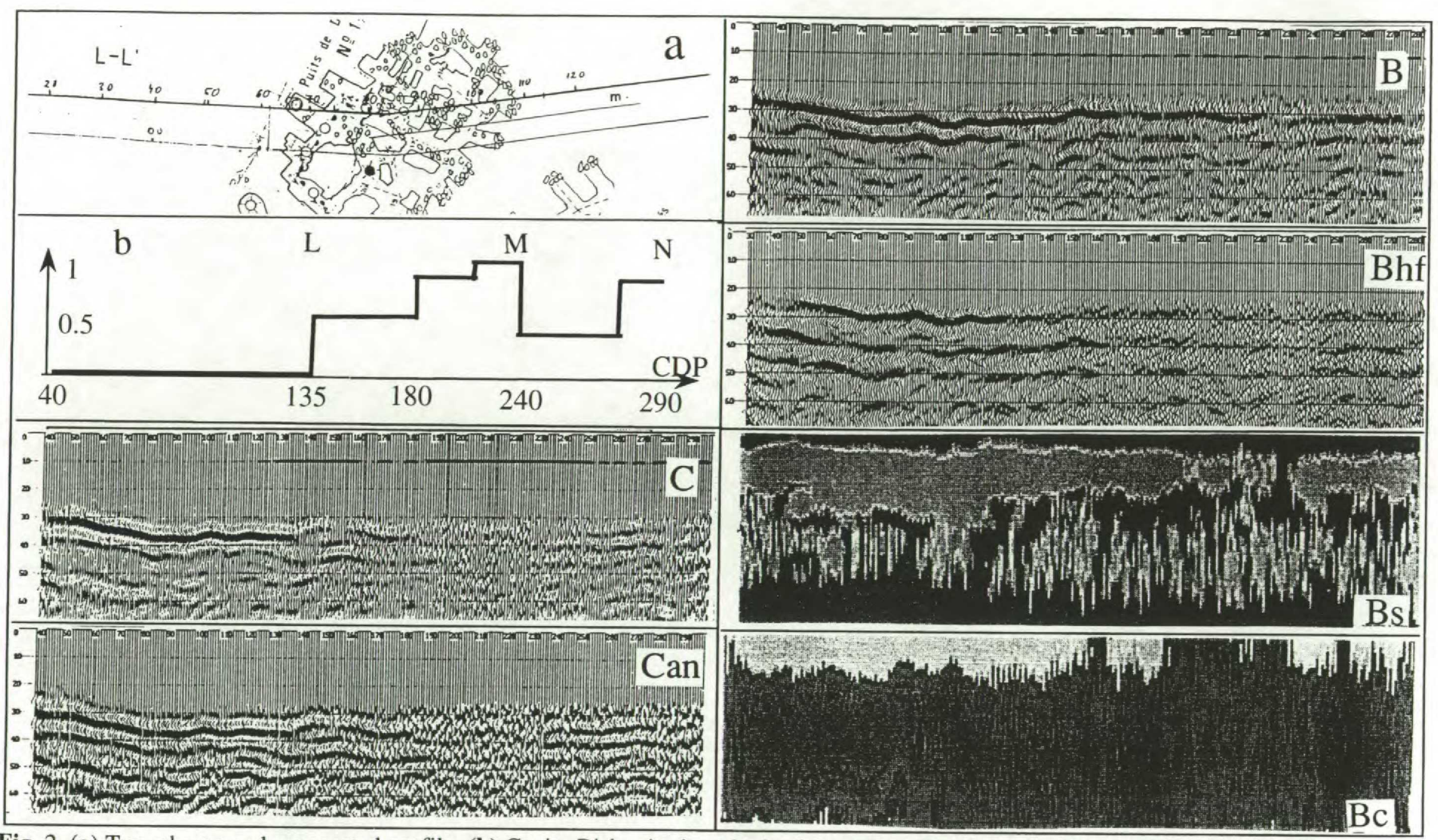

Fig. 2. (a) Tunnel map and prospected profile; (b) Cavity Risk criterion obtained ( tunnes are confirmed within zone L-M and are detected within zone M-N); (C) seismic section showing marker C: masking of this marker, well correlated with the tunnel positions is observed ; (Can) Bhf taken inside temporal window corresponding to the reflection occurence; (Bc) cross -correlation of the neighbouring traces of Bhf. 


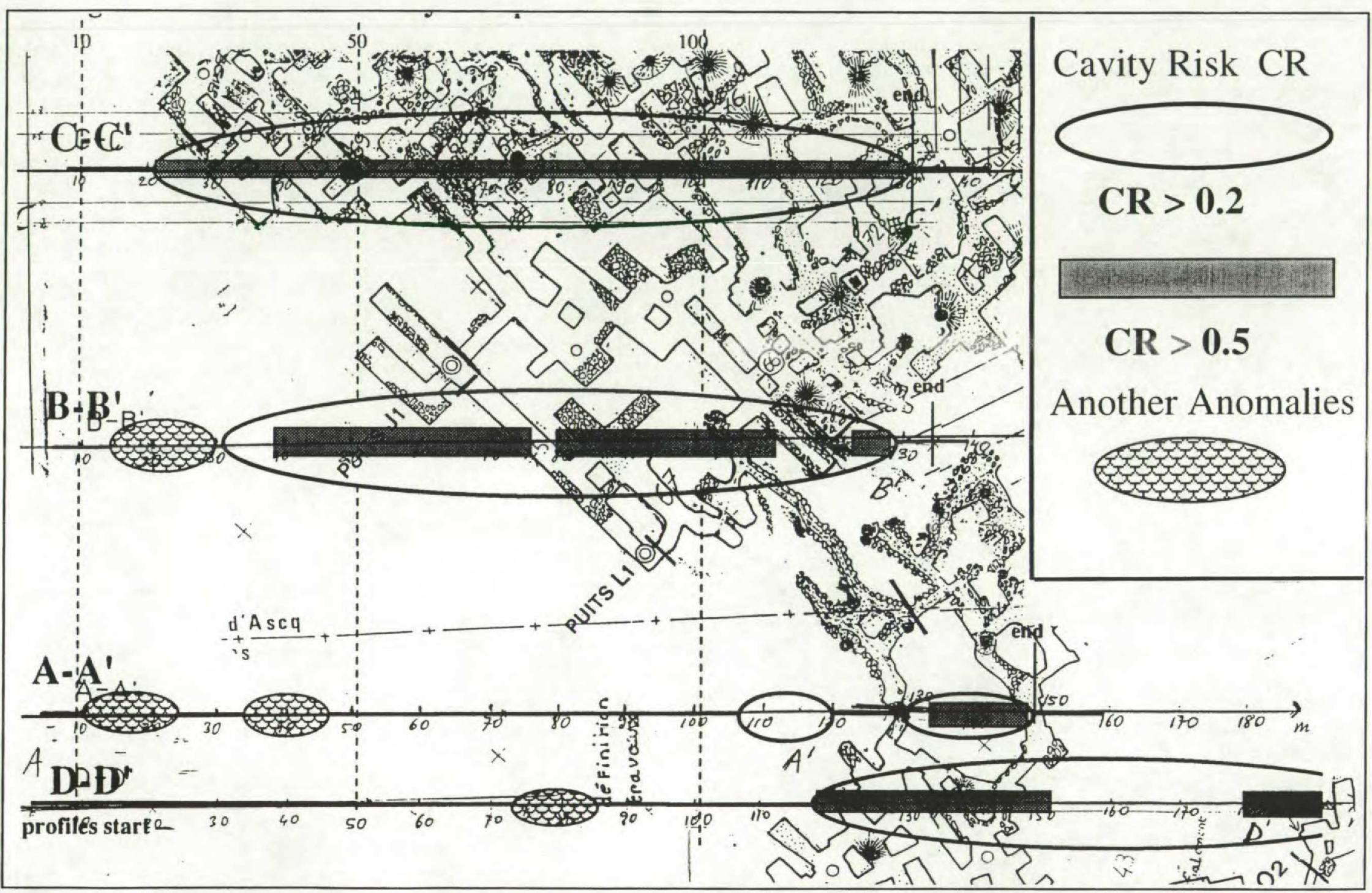

Fig. 3. Test for the method reliability performed along profiles A-A', B-B', C-C', D-D' . The obtained Cavity Risk criterion is superimposed over the tunnel map (communicated after detection). All prospected tunnels are detected, no tunel is omitted. 